\title{
A simple method for the construction of electrode arrays
}

\author{
A.J. Verloop and J. Holsheimer \\ Bio-information Group, Department of Electrical Engineering, Twente University of Technology, Enschede \\ (The Netherlands) \\ (Received January 24th, 1984) \\ (Revised June Sth, 1984) \\ (Accepted June 26th, 1984)
}

Key words: electrode array - recording electrode - insulated wire - field potential - in vitro brain slice

A simple method is described for the construction of electrode arrays consisting of insulated metal wires (33 $\mu \mathrm{m}$ diameter) spaced at small, equal distances $(0.1 \mathrm{~mm})$. No specialized instrumentation and techniques are needed, as only simple mechanical tools are sufficient. The electrode arrays are used for field potential recording from in vitro brain slice preparations.

\section{Introduction}

The recording of the spatio-temporal distribution of field potentials and the subsequent calculation of the current source density is a method of still increasing interest for the investigation of the properties and interactions of neuronal populations (Nicholson and Freeman, 1975; Mitzdorf and Singer, 1977; Leung, 1979; Rappelsberger et al., 1981). When using this method the simultaneous recording of field potentials at several positions has some advantages. One can be sure that all measurements are made under the same experimental conditions and it takes a fraction of the time required for making a sequence of recordings at different positions. Therefore arrays of equidistant electrodes have been developed for superficial and depth recording. During the last decade the construction of many of these multiple electrodes for in vivo and in vitro measurements is based on thin film techniques (Wise and Angell, 1975; Pickard, 1979; Pochay et al., 1979; Prohaska et al., 1979; Kuperstein and Whittington, 1981).

Since these integrated-circuit technologies are not available in or near most physiological laboratories, there is still a need for techniques to manufacture

Correspondence: J. Holsheimer, Bio-information Group, Department of Electrical Engineering, Twente University of Technology, P.O. Box 217, 7500 AE Enschede, The Netherlands. 
equidistant electrode arrays by relatively simple methods. Therefore a technique was developed for the construction of an electrode array, in which only simple mechanical tools are needed. This array consists of wire electrodes, kept in place by an insulating material. Various epoxies are available for insulation, but the problem is to keep a number of wires before and during the polymerization of the epoxy in their original position. A solution to this main problem is described. Using this technique, an array of 8 metal wire electrodes with diameters of $33 \mu \mathrm{m}$ and at a distance of 0.1 $\mathrm{mm}$ from each other was constructed for the recording of field potentials from hippocampal in vitro slices (Holsheimer, 1983).

\section{Materials and Methods}

The electrode material used, is insulated Karma wire with a diameter of $33 \mu \mathrm{m}$ (core diameter $25 \mu \mathrm{m}$ ) (California Fine Wire Co., Grover City, CA). The concept of positioning a number of wires in parallel at equal distances is based on the use of two springs, made of wire with a diameter which equals the distance between the electrodes. The springs are made of steel wire (diameter $0.1 \mathrm{~mm}$ ) and have an inner diameter of $1.0 \mathrm{~mm}$. Each spring is kept in position by a bar with a diameter of 0.5

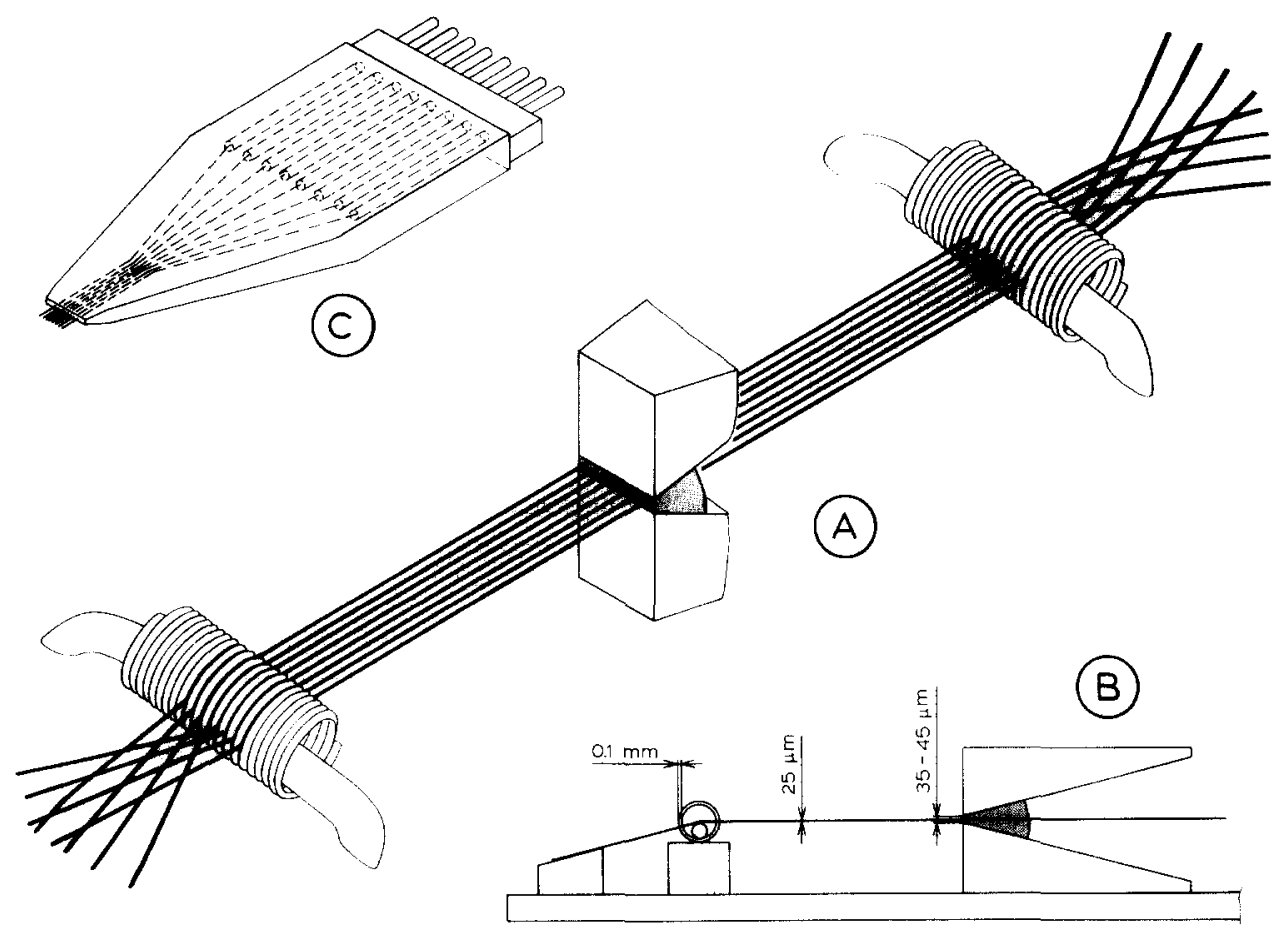

Fig. 1. Set-up for the construction of the electrode array. A: overall view of the principal parts (without mounting plate). B: side view of part of the set-up with mounting plate. $\mathrm{C}$ : complete electrode array with connector to input amplifiers. For details see text. 
mm (Fig. 1B). After stretching the springs somewhat, straight pieces of insulated Karma wire are placed between windings of the two springs and attached at both ends. Then, in the unstretched position, the springs retain the wires and determine the distance between the wires. The set-up is shown in Fig. 1A, B.

The next step is to place a mould in position and to fill it with epoxy. A teflon mould was made, which consists of two parts. This material does not become attached to epoxy. The lower part of the mould is placed under the array of wires between the two springs, leaving a space of 5-10 $\mu \mathrm{m}$ (Fig. 1B). Next the upper part of the teflon mould is placed exactly on top of the lower part, also leaving a space of $5-10 \mu \mathrm{m}$ from the wires. Then an epoxy (Hysol, resin C8-W795, hardener H-W796, Hysol Div., The Dexter Corp., Oleon, NY) is introduced between the two parts of the mould. The epoxy fills the mould by capillary force up to the end where the wires are close to its edges. The epoxy is polymerized at $60^{\circ} \mathrm{C}$ in air with a relative humidity of less than $20 \%$ during $1 \mathrm{~h}$. The parts of the mould are then removed and the electrode array can be completed.

On the front side the insulated wires are cut some milimetres from the epoxy tip. At the other end each wire is glued to a connector using a conductive paste (Dupont air-dry silver-4922). A second teflon mould is used to fill the area between the connector and the first epoxy tip with the same epoxy. The final step is to cut the wires carefully with a scalpel blade under visual inspection through a microscope. All wires are cut to the same length $(1-1.5 \mathrm{~mm})$ from the epoxy tip, so that the electrode tips make up a straight line of equidistant recording areas (Fig. 1C).

\section{Results}

An S.E.M. study of the electrode tips has revealed that hardly any deformation is produced by cutting the wires with a scalpel blade. The cores remain circular with the insulation close around the tips. The accuracy of the tip intervals is within $7.5 \%$.

The electrodes, with an impedance of $4-8 \mathrm{M} \Omega$ at $1 \mathrm{kHz}$, are used in combination with high impedance preamplifiers (Analog Devices, AD 515 or AD 545; R $=10^{15}$ $\Omega, \mathrm{C}=0.8 \mathrm{pF}$ ). The recording characteristics of the eight channels differ at most $2 \%$ at frequencies up to $250 \mathrm{~Hz}$ (low cut-off frequency $0.5 \mathrm{~Hz}$ ). These differences increase slightly to at most $4 \%$ at $2 \mathrm{kHz}$. Thus for the interesting range (up to 400 $\mathrm{Hz}$ ) the channels can be considered electrically equivalent.

The electrode noise has a mean value of $8.5 \mu \mathrm{V}$ (eff) within the frequency band of $0.5 \mathrm{~Hz}-5 \mathrm{kHz}$. Cross-talk between neighbouring channels is approximately $3 \%$ at $100 \mathrm{~Hz}$ and approximately $4 \%$ at $1 \mathrm{kHz}$. Cross-talk between non-neighbouring channels is less than $1 \%$ at both frequencies.

Recordings from guinea pig hippocampal slices $(0.6 \mathrm{~mm}$ thick) by means of an array of eight electrodes at intervals of $0.1 \mathrm{~mm}$ are shown in Fig. 2. These recordings were made in the CA1 field, 20-30 $\mu \mathrm{m}$ under the surface of the slice, with the array perpendicular to the laminated structure (Fig. 2B). Only electrode 8 was not in the CAl field, but in the dentate area. In Fig. 2A the averaged evoked responses to stimulation of Schaffer collaterals (at the level of electrode 5) are shown. The traces 

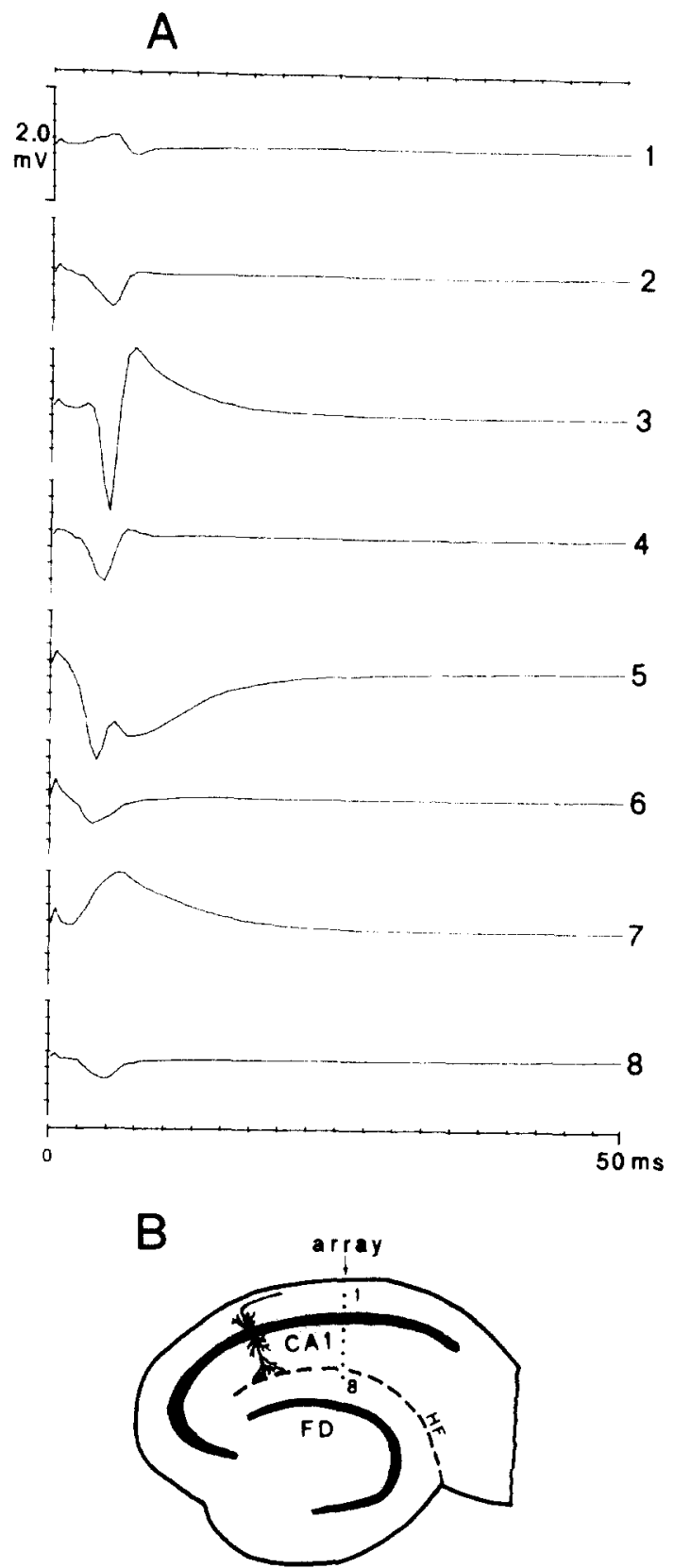

Fig. 2. Simultaneous field potential recordings of the CA1 field of the hippocampal slice preparation with an array of 8 electrodes at spacings of $0.1 \mathrm{~mm}$, perpendicular to the laminated structure. A: averaged evoked responses to stimulation of Schaffer collaterals; positive upward. B: position of the electrode array; electrodes $1-7$ in CA1 field, electrode 8 in dentate area (FD). HF, hippocampal fissure. 
1-8 correspond to the electrodes in Fig. $2 \mathrm{~B}$. The maximum of the (negative) synaptic response is at electrode 5 in the stratum radiatum and the maximum of the population spike is at electrode 3 in the stratum pyramidale.

\section{Discussion}

The construction of a multi-electrode as described in this paper is a simple method to obtain an equidistant array of recording tips. The accuracy of the tip spacings will depend on the precision of the finishing. With this method the number of electrodes, the electrode diameter and the spacing can be varied. Decreasing the electrode diameter and/or the interval will result in increased cross-talk between the channels. Since this array is constructed primarily for field potential recording of neuronal populations (not single cells), cross-talk will be of little significance, because this type of signal is restricted to a frequency range with an upper limit of $200-300 \mathrm{~Hz}$.

The same principle can also be used for the construction of a multiple depth electrode, incorporated in a hypodermic needle (cf. Barna et al., 1981). For this application the epoxy with the electrode array can be positioned in a side window in the needle and the electrode wires should be cut at the surface of the epoxy.

\section{Acknowledgement}

This work was supported by CLEO (National Committee for Epilepsy Research), Grant A-33.

\section{References}

Barna, J.S., Arezzo, J.C. and Vaughan, H.G. (1981) A new multi-electrode array for the simultaneous recording of field potentials and unit activity, Electroenceph. clin. Neurophysiol., 52: 494-496.

Holsheimer, J. (1983) Spatial properties of epileptiform discharges in hippocampal slices, Neurosci. Lett., Suppl. 14: S167.

Kuperstein, M. and Whittington, D.A. (1981) A practical 24 channel microelectrode for neural recording in vivo, IEEE Trans. Biomed. Eng., BME-28: 288-293.

Leung, L.S. (1979) Potentials evoked by alvear tract in hippocampal CA1 region of rats. II. Spatial field analysis, J. Neurophysiol., 42: 1571-1589.

Mitzdorf, U, and Singer, W. (1977) Laminar segregation of afferents to lateral geniculate nucleus of the cat. An analysis of current source density, J. Neurophysiol., 40: 1227-1244.

Nicholson, Ch. and Freeman, J.A. (1975) Theory of current source density analysis and determination of conductivity tensor for anuran cerebellum, J. Neurophysiol., 38: 356-368.

Pickard, R.S. (1979) A review of printed circuit microelectrodes and their production, J. Neurosci. Meth., 1: $301-318$.

Pochay, Ph., Wise, K.D., Allard, L.F. and Rutledge, L.T. (1979) A multichannel depth probe fabricated using electron-beam lithography, IEEE Trans. Biomed. Eng., BME-26: 199-206.

Prohaska, O., Pacha, F., Pfundner, P. and Petsche, H. (1979) A 16-fold semi-microelectrode for intracortical recording of field potentials, Electroenceph. clin. Neurophysiol., 47: 629-631. 
Rappelsberger, P.. Pockberger, H. and Petsche. H. (1981) Current source density analysis: methods and application to simultaneously recorded field potentials of the rabbit's visual cortex, Pflügers Archiv., 389: 159-170.

Wise, K.D. and Angell, J.B. (1975) A low-capacitance multielectrode probe for use in extracellular neurophysiology, IEEE Trans. Biomed. Eng., BME-22: 212-219. 